\title{
Impact of Pre-Dialysis Care on Clinical Outcomes in Peritoneal Dialysis Patients
}

\author{
Dandara N. Spigolon ${ }^{a}$ Thyago P. de Moraes ${ }^{a}$ Ana E. Figueiredo ${ }^{b}$ \\ Ana Paula Modesto ${ }^{a}$ Pasqual Barretti ${ }^{c}$ Marcus Gomes Bastos ${ }^{d}$ \\ Daniela V. Barreto $^{\mathrm{a}}$ Roberto Pecoits-Filho ${ }^{\mathrm{a}}$ on behalf of BRAZPD Investigators \\ a School of Medicine, Pontifícia Universidade Católica do Paraná (PUCPR), Curitiba, ${ }^{b}$ Pontifícia Universidade Católica \\ do Rio Grande do Sul (PUCRS), Porto Alegre, ${ }^{C}$ Universidade Estadual Paulista - UNESP, Botucatu, and ${ }^{\text {dUniversidade }}$ \\ Federal de Juiz de Fora - UFJF, Juiz de Fora, Brazil
}

\section{Key Words}

Clinical outcomes · Peritoneal dialysis · Pre-dialysis

care $\cdot$ Survival

\begin{abstract}
Background: Structured pre-dialysis care is associated with an increase in peritoneal dialysis (PD) utilization, but not with peritonitis risk, technical and patient survival. This study aimed at analyzing the impact of pre-dialysis care on these outcomes. Methods: All incident patients starting PD between 2004 and 2011 in a Brazilian prospective cohort were included in this analysis. Patients were divided into 2 groups: early pre-dialysis care ( 90 days of follow-up by a nephrology team); and late pre-dialysis care (absent or less than 90 days follow-up). The socio-demographic, clinical and biochemical characteristics between the 2 groups were compared. Risk factors for the time to the first peritonitis episode, technique failure and mortality based on Cox proportional hazards models. Results: Four thousand one hundred seven patients were included. Patients with early pre-dialysis care presented differences in gender (female -47.0 vs. $51.1 \%, p=0.01$ ); race (white -63.8 vs. $71.7 \%, p<0.01$ ); education ( $<4$ years 61.9 vs. $71.0 \%, p<0.01$ ), respectively, compared to late care.
\end{abstract}

Patients with early pre-dialysis care presented a higher prevalence of comorbidities, lower levels of creatinine, phosphorus, and glucose with a significantly better control of hemoglobin and potassium serum levels. There was no impact of pre-dialysis care on peritonitis rates (hazard ratio (HR) 0.88; 95\% $\mathrm{Cl} 0.77-1.01$ ) and technique survival (HR 1.12; 95\% Cl 0.92-1.36). Patient survival (HR 1.20; 95\% Cl 1.03-1.41) was better in the early pre-dialysis care group. Conclusion: Earlier pre-dialysis care was associated with improved patient survival, but did not influence time to the first peritonitis nor technique survival in this national PD cohort.

(c) 2016 S. Karger AG, Basel

\section{Introduction}

The number of patients with chronic kidney disease category G5 (CKD-5) starting renal replacement therapy (RRT) is increasing worldwide [1], and this is a reflection of the ageing and increasing incidence of risk factors for CKD (i.e. diabetes and hypertension) in the general population and increased access to RRT. Unfortunately, CKD is usually detected much later in the course of the disease due to the silent evolution and lack of screening pro-

\section{KARGER}

E-Mail karger@karger.com

www.karger.com/ajn
(C) 2016 S. Karger AG, Basel

0250-8095/16/0432-0104\$39.50/0
Roberto Pecoits-Filho, MD, PhD

School of Medicine, Pontifícia Universidade Católica do Paraná

Rua Imaculada Conceição, 1155

Curitiba, PR 80215-901 (Brazil)

E-Mail r.pecoits@pucpr.br 
grams. Therefore, CKD disease-management programs should be developed and are of an utmost necessity [1]. This is particularly evident in many countries, where there is increasing access to RRT and no structured CKD care programs [2].

CKD patients in hemodialysis (HD) and peritoneal dialysis (PD) are at higher risks of hospitalization and mortality [3]. In PD, understanding factors associated with high mortality and technique failure (particularly due to peritonitis) rates is a major challenge in the management of this population group $[2,3]$. Adequate CKD management is associated with better patient survival in previous reports in the HD population $[4,5]$. In addition, previous studies described that patients with adequate pre-dialytic care presented better control of anemia, nutritional status and cardiovascular (CV) condition $[5,6]$. Interestingly, structured pre-dialysis care was associated with an increase in patient choice of PD and increase in the penetration of PD use [7-9].

Studies looking broadly at the impact of pre-dialysis care on outcomes (patient and technique survival), particularly in centers with fast expansion of access to dialysis treatment are scarce and limited to HD, except for one single-center study from China [10]. Finally, an analysis on the impact of pre-dialysis care on patient outcomes adjusted for laboratory, nutritional and hydroelectrolytic imbalance has not been performed until the present. In this study, we aimed at analyzing the impact of pre-dialysis care on PD patients and technique outcomes in a Brazilian national representative cohort.

\section{Methods}

\section{Patient Population}

This is a prospective study based on the BRAZPD II cohort, including data from 122 dialysis centers of all regions from Brazil. The number of prevalent patients in each year corresponded to $65-70 \%$ of all PD patients in the country in that same year. Data collection included socio-demographic data and comorbid conditions at the baseline and various prescription, clinical and laboratory data, collected periodically depending on the variable. The main outcomes captured in the study were dropped out from PD and total and cause-specific mortality [11].

For this study, we identified all adult patients starting PD between 2004 and 2011, and within that subset, all those with valid data about pre-dialysis care were included. Patients with previous RRT were excluded (fig. 1). All patients provided written consent, which was approved by the Brazilian Ethical Committee number 448. For the comparative analysis, patients were stratified into 2 groups according to the time of pre-dialysis care: patients who had at least 90 days of pre-dialysis follow-up with a nephrology team (early pre-dialysis care) and patients who did not have or had

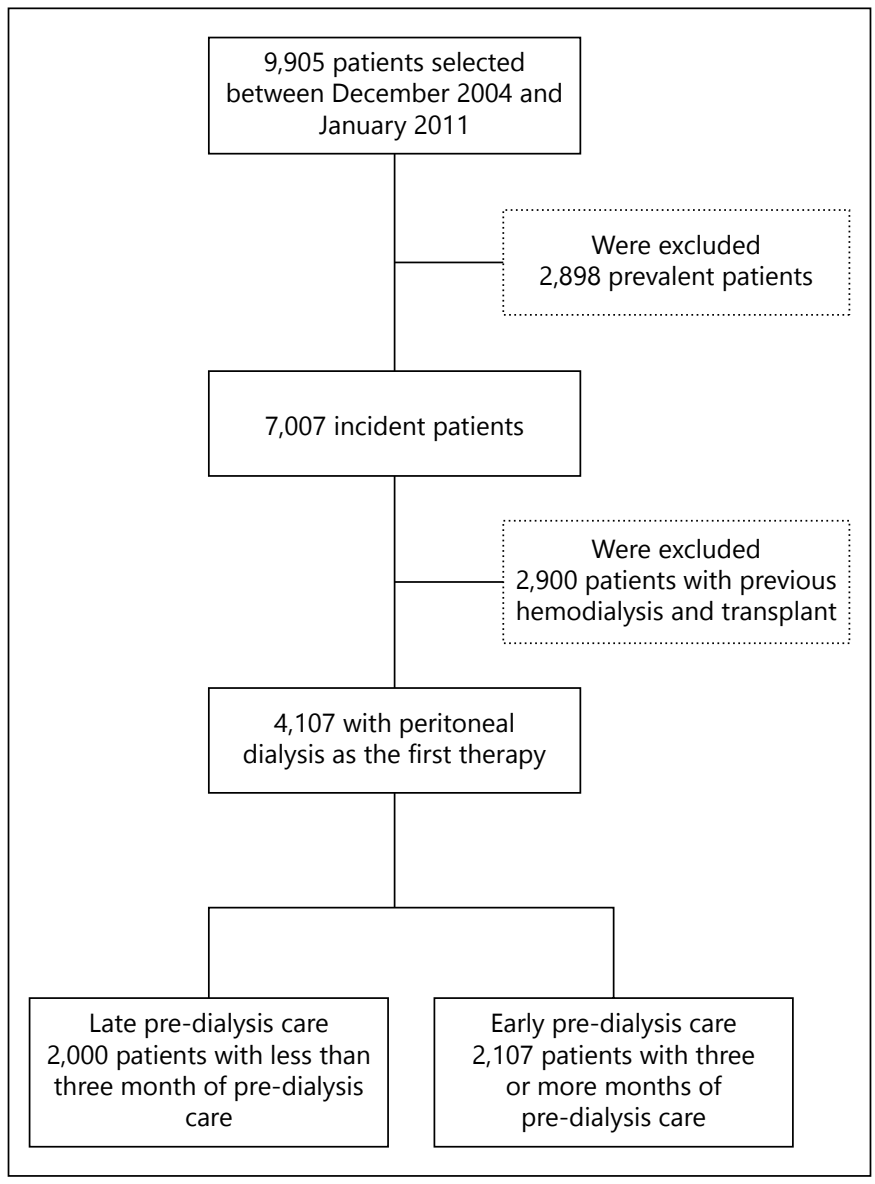

Fig. 1. Flowchart of the study design and patient selection.

less than 90 days follow-up with a nephrology team (late pre-dialysis care). Center experience was measured in patient-year, for example, the follow-up time of all patients from a certain center was summed up and the result divided by the number of years that the center participated in the study.

\section{Data Collection}

Data were collected using PDNet software that contained a form with important and relevant information for the design of the study. The pre-dialysis questionnaire was included in the medical form, providing information that characterized the pre-dialysis nephrological care (i.e. whether the patient received pre-dialysis care, time that received care, if received orientation regarding dialysis method options, how the treatment was indicated, by whom was the patient referred to a nephrology specialist and if the specialist had vascular access). The pre-dialysis medical questionnaire, and 2 questions ' (1) Did the patient receive pre-dialysis care? and (2) For how long was the pre-dialytic care done?' were used to define the groups for the analysis.

\section{Clinical Outcomes}

Death, recovery of renal function, renal transplantation, permanent transfer to $\mathrm{HD}$, and lost to follow-up were factors based on which dropouts were defined. In the analysis of time to first 
peritonitis episode, those who dropped out of the study before experiencing any peritonitis episode and those were still alive at the end of follow-up without any peritonitis episode were censored. In the analysis of technique survival, the event of interest was defined as permanent switch to HD because of any valid cause. Patients who died, those who were still alive at the end of the follow-up, and those who received a renal transplantation were censored. For the analysis of patient survival, the event of interest was death by any cause, and all patients who dropped out of the study for any reason or those still alive at the end of the study were censored.

\section{Statistical Analysis}

Continuous variables were expressed as mean $\pm \mathrm{SD}$ or median and range, while categorical variables (e.g. gender, race, primary renal disease, presence of comorbid conditions, initial therapy, current PD modality, etc.) were expressed as frequencies or percentages. The traditional Cox proportional hazards model was used for clinical outcomes, and to be included in the multivariate model, a covariate should have had a p value $<0.20$ in the univariate analysis. Cox proportional hazard models were estimated using SPSS 20.0. Assumptions for proportional hazards were checked with residual plots. Statistical significance was set at the level of $\mathrm{p}<$ 0.05 . Two models were constructed to analyze the independent effect of pre-dialysis care on patient outcome: the first model analyzed only demographic and clinical variables, while the second model also included biochemical variables (hemoglobin, potassium and phosphorus).

\section{Results}

\section{Patient Characteristics}

The flowchart of patients selected for the analysis is summarized in figure 1 . Among the 4,107 incident patients on $\mathrm{PD}, 2,107$ patients presented early and 2,000 patients presented late for pre-dialysis care. Clinical and demographical characteristics of the study population are presented in table 1. In general, patients from the early pre-dialysis care group present lower educational levels (61.9\% with less than 4 years of formal education vs. $71.0 \%)$ and exhibited a significant higher prevalence of comorbidities: coronary (23.4 vs. 19.5 ), diabetes (50.0 vs. $39.8 \%)$, hypertension (75.6 vs. $64.3 \%)$, left ventricular hypertrophy (30.1 vs. $20.1 \%)$, peripheral artery diseases (19.9 vs. $16.2 \%$ ), and overweight ( $45.8 \%$ with a body mass index (BMI) $>25$ vs. 40.9). Finally, patients with early predialysis care tended to have lower average levels of creatinine, phosphorus, glucose and higher levels of hemoglobin at the first 3 months of PD.

The dynamics of biochemical variables analyzed throughout the subsequent quarters of the first year on PD treatment showed that although phosphorus control were not significantly different between the groups, pa- tients from the early pre-dialysis care presented a significantly better control of hemoglobin and potassium serum levels (table 1 and fig. 2).

\section{Time to First Peritonitis Episode}

The percentage of patients without pre-dialysis care who presented at least one peritonitis episode was 12.8 $(\mathrm{n}=257)$, while in the group with pre-dialysis care the percentage was $15.6(\mathrm{n}=329)$. The time to the first peritonitis episode was similar between groups even after adjustment for covariates (hazard ratio (HR) 0.88 ; 95\% CI $0.77-1.01)$. There were only 2 independent predictors for the primary event of interest: cancer (HR 1.48; 95\% CI 1.07-2.02) and education $<4$ years (HR 1.17; 95\% CI 1.01-1.35). The multivariate model comprised the following variables: dialysis vintage, BMI, cancer, center experience, education level and region of the country. Again, the inclusion of biochemical variables did not alter the effect of pre-dialysis care in the outcome.

\section{Technique Failure}

Early pre-dialysis care was not significantly associated with technique failure (HR 1.12; 95\% CI 0.92-1.36). In absolute numbers, there were $242(11.5 \%)$ dropouts in the group with pre-dialysis careand 199 (9.9\%) in those without pre-dialysis care. On the basis of the criteria described above, we included the following covariates in the univariate analysis: cancer, coronary artery disease, center experience, diabetes, distance to the PD center, gender, PD modality, region of the country and peripheral artery disease. The covariates that were significantly associated with technique failure in the multivariate analysis were center experience (per 1 patient-year of increase: HR 0.99; 95\% CI 0.98-0.99), female gender (HR 0.79; 95\% CI 0.65-0.96) and continuous ambulatory PD (CAPD) (HR 0.82; 95\% CI 0.67-0.99).

\section{Patient Survival}

The main cause of death was CV disease for the late pre-dialysis group with 142 events (40.9\%), followed by sepsis not related to PD with 101 events (29.1\%), while in the early pre-dialysis group it was the inverse: sepsis with 154 events (40.8\%) and CV disease with 127 events (33.7\%). The univariate analysis identified age $>65$ years, BMI, cancer, coronary artery disease, peripheral artery disease, center experience, diabetes, education $<4$ years, hypertension, PD modality and race as significantly associated with the primary event of interest. Late pre-dialysis care (HR 1.20; 95\% CI 1.03-1.41), age $>65$ years (HR $2.97 ; 95 \%$ CI $2.52-3.50$ ), BMI $<18.5 \mathrm{~kg} / \mathrm{m}^{2}$ (HR 1.58 ; $95 \%$ CI 1.22-2.05), diabetes (HR 1.45; 95\% CI 1.24-1.69), edu- 
Table 1. Baseline characteristics of the studied population divided according to pre-dialysis care time of followup

\begin{tabular}{|c|c|c|c|}
\hline Variables & $\begin{array}{l}\text { Early pre-dialysis } \\
\text { care }(\mathrm{n}=2,107)\end{array}$ & $\begin{array}{l}\text { Late pre-dialysis } \\
\text { care }(n=2,000)\end{array}$ & $\mathrm{p}$ value \\
\hline \multicolumn{4}{|l|}{ Sociodemographic } \\
\hline Age $>65$ years, $\%$ & 43.7 & 43.1 & 0.73 \\
\hline Age, years & $61.1 \pm 16.2$ & $61.1 \pm 16.3$ & 0.99 \\
\hline Gender, female, $\%$ & 47.0 & 51.1 & 0.01 \\
\hline Race (white), \% & 63.8 & 71.7 & $<0.01$ \\
\hline Modality (CAPD), \% & 53.9 & 52.9 & 0.56 \\
\hline Center experience (patients-year) & $42.6 \pm 25.5$ & $47.1 \pm 25.6$ & $<0.01$ \\
\hline Distance to PD center $<25 \mathrm{~km}, \%$ & 77.4 & 78.8 & 0.38 \\
\hline Education $<4$ years, $\%$ & 61.9 & 71.0 & $<0.01$ \\
\hline Family income $<2 \mathrm{MW}, \%$ & 68.4 & 66.6 & 0.23 \\
\hline \multicolumn{4}{|l|}{ Comorbidities } \\
\hline Coronary artery disease (yes), $\%$ & 23.4 & 19.5 & $<0.01$ \\
\hline Diabetes (yes), $\%$ & 50.0 & 39.8 & $<0.01$ \\
\hline Hypertension (yes), \% & 75.6 & 64.3 & $<0.01$ \\
\hline Left ventricular hypertrophy (yes), \% & 30.1 & 20.1 & $<0.01$ \\
\hline Malignancy (yes), \% & 2.5 & 3.5 & 0.07 \\
\hline Peripheral artery disease (yes), \% & 19.9 & 16.2 & $<0.01$ \\
\hline DBP - 1st quarter, $\mathrm{mm} \mathrm{Hg}$ & $81.5 \pm 11.1$ & $81.9 \pm 12.1$ & 0.20 \\
\hline SBP - 1st quarter, $\mathrm{mm} \mathrm{Hg}$ & $136.3 \pm 20.1$ & $135.3 \pm 21.3$ & 0.12 \\
\hline BMI & $24.5 \pm 4.5$ & $25.1 \pm 4.7$ & $<0.01$ \\
\hline BMI, $\mathrm{kg} / \mathrm{m}^{2}, \%$ & & & $<0.01$ \\
\hline$<18.5$ & 5.5 & 7.4 & \\
\hline $18.5-25$ & 48.6 & 51.7 & \\
\hline$>25$ & 45.8 & 40.9 & \\
\hline \multicolumn{4}{|l|}{ Laboratory } \\
\hline Creatinine - 1 st quarter, $\mathrm{mg} / \mathrm{dl}$ & $6.0 \pm 2.6$ & $6.3 \pm 3.1$ & $<0.01$ \\
\hline Glucose - 1st quarter, mg/dl & $125.5 \pm 56.1$ & $129.6 \pm 58.3$ & 0.03 \\
\hline Hemoglobin - 1st quarter, g/dl & $11.0 \pm 1.7$ & $10.7 \pm 1.8$ & $<0.01$ \\
\hline Hemoglobin, g/dl, \% & & & $<0.01$ \\
\hline$<10$ & 25.8 & 31.9 & \\
\hline $10-12$ & 46.3 & 45.4 & \\
\hline$>12$ & 27.9 & 22.7 & \\
\hline Phosphorus - 1st quarter, mg/dl & $4.86 \pm 1.2$ & $4.93 \pm 1.3$ & 0.07 \\
\hline Phosphorus, g/dl, \% & & & 0.62 \\
\hline$<3.5$ & 49.6 & 48.9 & \\
\hline $3.5-5.0$ & 10.9 & 10.3 & \\
\hline$>5.5$ & 39.5 & 40.8 & \\
\hline Potassium - 1st quarter, mEq/l & $4.4 \pm 0.7$ & $4.5 \pm 0.8$ & 0.03 \\
\hline Potassium, $\mathrm{mEq} / \mathrm{l}, \%$ & & & $<0.01$ \\
\hline$>5.5$ & 5.2 & 8.8 & \\
\hline $3.5-5.5$ & 87.5 & 82.9 & \\
\hline$<3.5$ & 7.3 & 8.3 & \\
\hline
\end{tabular}

cation $<4$ years (HR 1.20; 95\% CI 1.01-1.43), diagnosis of hypertension at baseline (HR 0.77; 95\% CI 0.65-0.91), CAPD (HR 1.30; 95\% CI 1.11-1.52), year of initiation of dialysis (HR 1.50; 95\% CI 1.19-1.89 for those starting in 2005/2006 and HR 1.47; 95\% CI 1.18-1.83 for 2007/2008 compared to 2009/2010) and peripheral artery disease
(HR 1.40; 95\% CI 1.18-1.66) remained significantly associated with death in the multivariate analysis. The inclusion of laboratory values as covariates did not alter the impact of pre-dialysis care on mortality. Table 2 summarizes the effect of pre-dialysis care for all events studied, while figure 3 depicts the curves for time to event. 
Fig. 2. Multivariate cox regression analysis of predictors' pre-dialysis care as a predictor of first peritonitis episode, technique and patient survival.

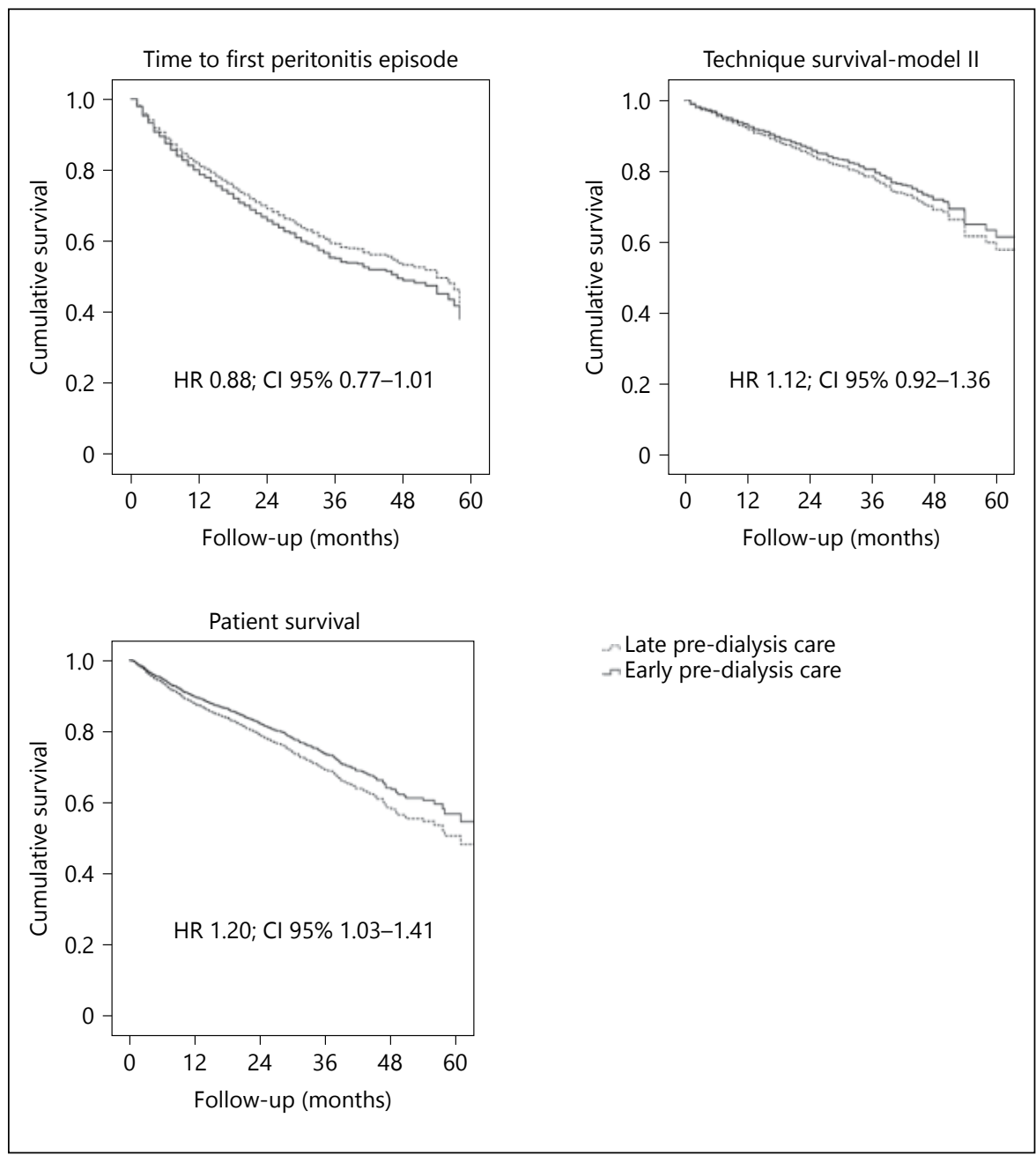

Table 2. Uni- and multivariate analyses of the impact of pre-dialysis care on clinical outcomes (detailed description of the variables is included in the online supplementary tables S1-S3 (for all online suppl. material, see www.karger.com/doi/10.1159/000444401)

\begin{tabular}{|c|c|c|c|c|c|c|}
\hline & Univariate & $\mathrm{p}$ value & $\begin{array}{l}\text { Multi-model I } \\
\text { demographic only }\end{array}$ & $\mathrm{p}$ value & $\begin{array}{l}\text { Multi-model II } \\
\text { demographic + laboratory }\end{array}$ & $\mathrm{p}$ value \\
\hline Time to first peritonitis episode & $1.11(0.97-1.27)$ & 0.14 & $0.90(0.78-1.03)$ & 0.13 & $0.88(0.77-1.01)$ & 0.08 \\
\hline Technique survival & $0.88(0.73-1.06)$ & 0.19 & $1.17(0.96-1.42)$ & 0.11 & $1.12(0.92-1.36)$ & 0.25 \\
\hline Patient survival & $1.25(1.08-1.45)$ & $<0.01$ & $1.27(1.09-1.48)$ & $<0.01$ & $1.20(1.03-1.41)$ & $<0.01$ \\
\hline
\end{tabular}

\section{Discussion}

To the best of our knowledge this is the first large cohort study to evaluate the impact of pre-dialysis care on PD patient outcomes, indicating that early pre-dialysis care is independently associated with better survival on $\mathrm{PD}$, even after adjusting for risk factors including bio- chemical variables. Moreover, pre-dialysis care was associated with an attenuation of CKD progression [8], better clinical outcome in HD patients and greater frequency of $\mathrm{PD}$ as the first choice in the RRT [12].

Early and late pre-dialysis groups differed significantly in some clinical and demographic characteristics (table 1). Not surprisingly, patients from the late pre-dialysis 
Fig. 3. Dynamics of hemoglobin, potassium and phosphorus normal range prevalence across the first year on PD divided according to the pre-dialysis care characteristics.

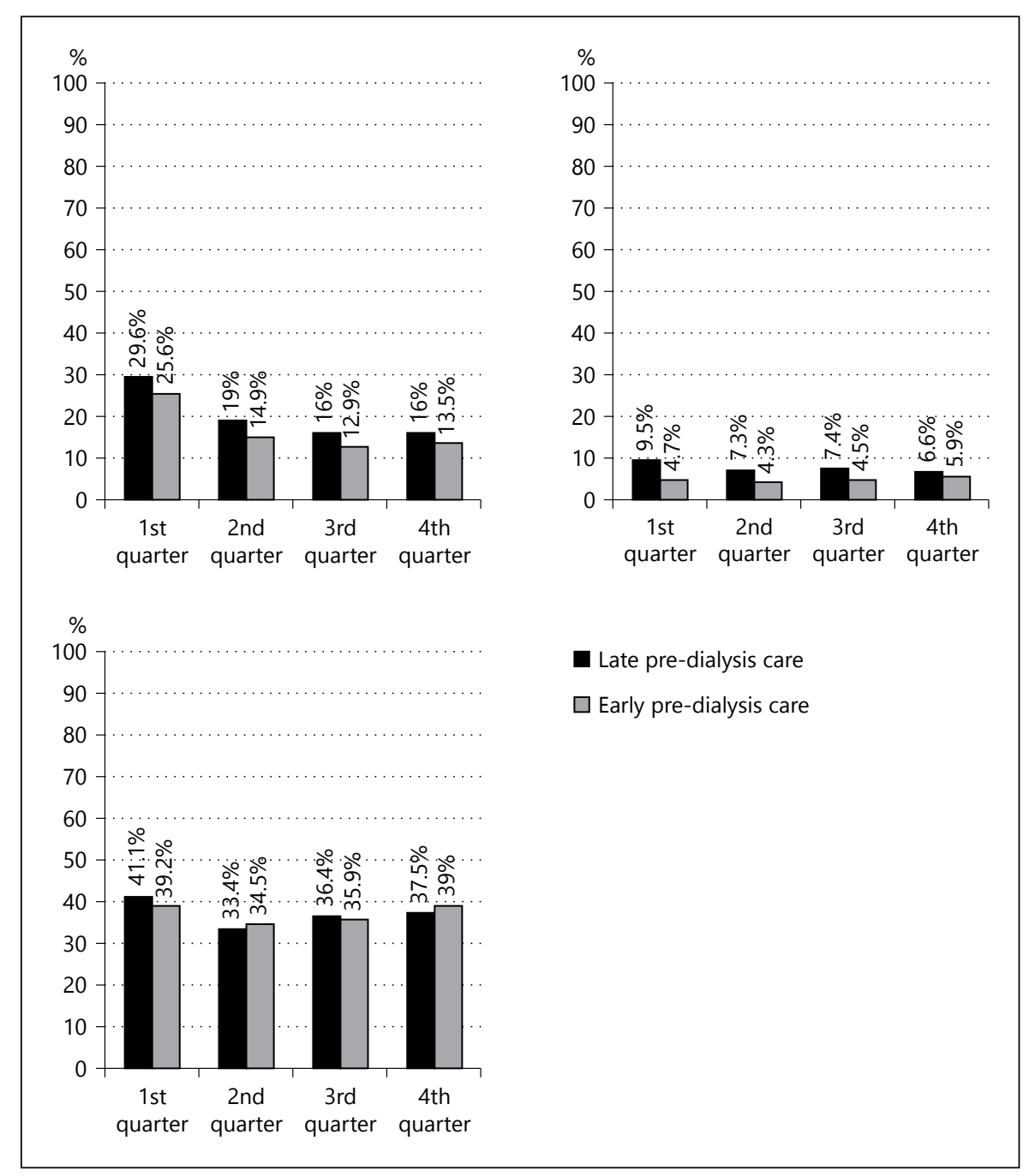

group had a lower education level, a lower prevalence of comorbidities and uncontrolled biochemical variables, including higher potassium and lower hemoglobin levels. This is probably due to earlier referral of these patients having comorbidities and are then assisted, different from the asymptomatic, many of whom do not know who have CKD and then are referred later to nephrologists. These negative effects of the lack of pre-dialysis care on biochemical parameters have already been described in the late nineties [13] and may play a significant role in patient outcomes.

Our study is the first attempt to analyze the possible impact of pre-dialysis care in technique survival and in the time to first peritonitis episode in PD patients. We found no association between pre-dialysis care and those outcomes. The lack of association between pre-dialysis care with technique failure could be explained by the fact

Impact of Pre-Dialysis Care on PD that previously identified risk factors for this outcome, such as membrane profile, dialysis vintage, sex, literacy, diabetes, glucose exposure, and anuria, cannot be modified by a more appropriate pre-dialysis care $[11,14,15]$. The same holds true for the time-to-first-peritonitis episode, since its most commonly described risk factors are diabetes, education, family income, region (e.g. rural or not), home distance to the PD center, age and race. More importantly, adequate dialysis care, including patient selection and training may be enough to offer patients adequate technique survival and risk of peritonitis, regardless of pre-dialysis care. Nevertheless, differences related to the pattern of training addressed to patients with and without pre-dialysis care may impact the time to first peritonitis outcome [16].

Importantly, we observed that early pre-dialysis care significantly affected patient survival even after adjust- 
ments for several and important covariates. One of the possible reasons to explain such differences in mortality rates is the worst control of biochemical/metabolic parameters in patients not receiving pre-dialysis care. In fact, a hemoglobin level $<10 \mathrm{~g} / \mathrm{dl}$, a potassium level either $<3.5$ or $>5.5 \mathrm{mEq} / \mathrm{l}$ and a phosphorus level $<3.5 \mathrm{mg} / \mathrm{dl}$ at baseline were significantly associated with mortality in the univariate analysis. Nevertheless, the inclusion of biochemical covariates at baseline in the model does not alter the negative impact of late pre-dialysis care. This may be partially explained because that difference initially observed at baseline disappears along the first year of treatment (fig. 2). Another possibility to explain these differences is changes in residual renal function (RRF), which are not captured in our study. However, since all patients were undergoing dialysis, with no history of previous HD or transplantation, it is unlikely that RRF differed significantly in the groups. Another interesting finding was the difference in the main cause of death between groups, which helps to raise a hypothesis for the underlying mechanism behind the higher mortality in those patients with late pre-dialysis care.

We observed that patients with late dialysis care died more frequently from CV disease. The absence of adequate pre-dialysis care may reflect less access to adequate implementation of CV prevention, particularly the management of traditional and (perhaps more importantly) non-traditional risk factors. In the early pre-dialysis group, the inverse predominated sepsis was compared to $\mathrm{CV}$ events; this perhaps justifies a greater control of risk for $\mathrm{CV}$ events due to early pre-dialysis care, accounting for the highest incidence for sepsis in this group.

Finally, the analysis of the dynamics of biochemical variables during the first months on PD treatment provides interesting insight about the impact of the transition between pre-dialysis to PD on the correction of the variables. In this study, while serum phosphorus levels were not significantly different between the groups divided according to pre-dialysis care during the first year of follow-up, patients with an early pre-dialysis care always presented significantly higher levels of hemoglobin and lower levels of potassium throughout the first 9 months on PD. Since these are important predictors of mortality in this population [17], the longer exposure to inadequate control of hemoglobin and potassium may at least in part justify the higher mortality observed in the group of patients with late pre-dialysis care.

However, the observation of the independent association between late pre-dialysis care and mortality, despite being corrected for those biochemical risk factors, indi- cates that other factors not captured in the present analysis may be involved. Using the same rationale, since patients with late pre-dialysis care who corrected their biochemical parameters were exposed to a high risk of mortality, a memory effect of the worst control of these variables may be carried to later phases on dialysis.

This study has some limitations: first is its observational design, which demands caution when interpreting all the results; second is the lack of information regarding known risk factors for both mortality and technique failure as RRF and membrane characteristics; last, details about specific characteristics of how pre-dialysis care was delivered, and particularly the eGFR at dialysis initiation were not captured in the present study.

However, our study presents important strengths: it is based on a national prospective cohort, adjustments were performed for several covariates and the general characteristics of our population are very similar with other large cohorts from different parts of the world.

In conclusion, we showed that longer pre-dialysis care provided a significant benefit for patient survival, but did not influence the time to the first peritonitis or technique survival in this national PD cohort. The reasons for such effects should be explored in future studies.

\section{Financial Disclosures}

Dr. R. Pecoits-Filho is a recipient of a scholarship from the Brazilian Council for Research (CNPq), received research grants. Dr. R. Pecoits-Filho, A.E. Figueiredo, P. Barretti and T.P. de Moraes received consulting fees and speaker honorarium from Baxter Healthcare. The other authors declare that they have no other relevant financial interests. This study (design, implementation, software development, data collection) was funded by Baxter Healthcare, Brazil. The current data extraction and analysis were supported by an investigator-driven study grant provided to PUCPR, as part of the Clinical Evidence Council Program from Baxter Healthcare.

References

1 Kidney Disease: Improving Global Outcomes (KDIGO) CKD-MBD Work Group: KDIGO clinical practice guideline for the diagnosis, evaluation, prevention, and treatment of chronic kidney disease-mineral and bone disorder (CKD-MBD). Kidney Int Suppl 2009; 113:S1-S130

2 Li PK, Szeto CC, Piraino B, et al: Peritoneal dialysis-related infections recommendations: 2010 update. Perit Dial Int 2010;30:393-423.

3 Fried LF, Bernardini J, Johnston JR, Piraino B: Peritonitis influences mortality in peritoneal dialysis patients. J Am Soc Nephrol 1996;7: 2176-2182. 
4 Singhal R, Hux JE, Alibhai SM, Oliver MJ: Inadequate predialysis care and mortality after initiation of renal replacement therapy. Kidney Int 2014;86:399-406.

5 Jungers P, Massy ZA, Nguyen-Khoa T, et al: Longer duration of predialysis nephrological care is associated with improved long-term survival of dialysis patients. Nephrol Dial Transplant 2001;16:2357-2364.

6 Kurella Tamura M, Li S, Chen SC, et al: Educational programs improve the preparation for dialysis and survival of patients with chronic kidney disease. Kidney Int 2014;85: 686-692.

7 Diaz-Buxo JA: The importance of pre-ESRD education and early nephrological care in peritoneal dialysis selection and outcome. Perit Dial Int 1998;18:363-365.

8 Chen YR, Yang Y, Wang SC, et al: Effectiveness of multidisciplinary care for chronic kidney disease in Taiwan: a 3-year prospective cohort study. Nephrol Dial Transplant 2013; 28:671-682.
9 Ribitsch W, Haditsch B, Otto R, et al: Effects of a pre-dialysis patient education program on the relative frequencies of dialysis modalities. Perit Dial Int 2013;33:367-371.

10 Chow KM, Szeto CC, Law MC, Kwan BC, Leung CB, Li PK: Impact of early nephrology referral on mortality and hospitalization in peritoneal dialysis patients. Perit Dial Int 2008;28:371-376.

11 Moraes TP, Figueiredo AE, de Campos LG, Olandoski M, Barretti P, Pecoits-Filho R: Characterization of the BRAZPD II cohort and description of trends in peritoneal dialysis outcome across time periods. Perit Dial Int 2014;34:714-723.

12 Abbott KC, Trespalacios FC, Taylor AJ, Agodoa LY: Atrial fibrillation in chronic dialysis patients in the United States: risk factors for hospitalization and mortality. BMC Nephrol 2003;4:1.

13 Wu IW, Wang SY, Hsu KH, et al: Multidisciplinary predialysis education decreases the incidence of dialysis and reduces mortality - a controlled cohort study based on the NKF/ DOQI guidelines. Nephrol Dial Transplant 2009;24:3426-3433.
14 de Moraes TP, Olandoski M, Caramori JC, et al: Novel predictors of peritonitis-related outcomes in the BRAZPD cohort. Perit Dial Int 2014;34:179-187.

15 Martin LC, Caramori JC, Fernandes N, et al: Geographic and educational factors and risk of the first peritonitis episode in Brazilian peritoneal dialysis study (BRAZPD) patients. Clin J Am Soc Nephrol 2011;6:19441951.

16 Figueiredo AE, Moraes TP, Bernardini J, et al: Impact of patient training patterns on peritonitis rates in a large national cohort study. Nephrol Dial Transplant 2015;30: 137-142.

17 Ribeiro SC, Figueiredo AE, Barretti P, Pecoits-Filho R, de Moraes TP: Low serum potassium levels increase the infectious-caused mortality in peritoneal dialysis patients: a propensity-matched score study. PLoS One 2015; 10:e0127453. 